\title{
Local structure of dielectric framework materials
}

\author{
Anthony Phillips ${ }^{1}$, Helen Duncan ${ }^{2}$, Martin Dove ${ }^{1}$, David Keen ${ }^{3}$, Matthew Tucker ${ }^{4}$ \\ ${ }^{1}$ School Of Physics And Astronomy, Queen Mary University Of London, London, United Kingdom, ${ }^{2}$ Centre for Science at Extreme \\ Conditions, University of Edinburgh, Edinburgh, United Kingdom, ${ }^{3}$ ISIS Neutron and Muon Source, Didcot, United Kingdom, \\ ${ }^{4}$ Spallation Neutron Source, Oak Ridge National Laboratory, Oak Ridge, TN, United States \\ E-mail: a.e.phillips@qmul.ac.uk
}

Many materials with important electrical properties owe these to the motion of guest ions within a relatively rigid framework. Such motion may, for instance, cause piezoelectricity, ferroelectricity, or ion sorption or conduction, leading to appliations in sensors, actuators, energy harvesters, capacitors, data storage, nuclear waste cleanup, or batteries. The best-studied materials of this sort have historically been inorganic compounds in which the moving ions are monoatomic metal cations: for instance, the perovskite and Prussian blue families have proven rich sources of electrical functionality. Recently, however, many new materials have been reported in which the guests are polyatomic organic cations [1]. Among the most famous examples are the methylammonium and formamidinium lead iodide perovskites, whose attractive optoelectronic properties have been widely studied for solar cell applications. But the family of hybrid electroactive framework materials is much broader than just these. The range of organic guests that can be included appears to be limited only by the size of the interstices, and includes substituted ammoniums, guanidinium, formamidinium, azetidinium, hydrazinium, and imidazolium ions.

The resulting hybrid materials often display the same properties that make their inorganic analogues so useful. Often, for instance, the organic cation is dynamically disordered at high temperatures, but on cooling freezes into favourable orientations. Such a phase transition will naturally cause a substantial decrease in the material's dielectric constant, and may also involve the onset of ferroelectric order. The intermolecular forces that give rise to these properties, however, are subtly different from those in their inorganic analogues. Unlike monoatomic metal cations, organic cations may have intrinsic dipole moments, or indeed higher-order multipole moments [2]. Furthermore, not just electrostatic interactions but also hydrogen bonding between guest and framework are critically important. Understanding structure-property relationships in this family well enough to enable crystal engineering will require a detailed understanding of both of these effects. Yet, even more so than in the inorganic parent materials, the disorder in high-temperature phases prevents traditional crystallographic methods from giving an accurate picture of the guest-framework interactions. We must turn instead to diffraction methods sensitive to local structure, such as total neutron scattering.

We present here total neutron scattering measurements from two representative materials in this family: the perovskite analogue dimethylammonium manganese formate, $\left(\mathrm{CH}_{3}\right)_{2} \mathrm{NH}_{2}\left[\mathrm{Mn}\left(\mathrm{HCO}_{2}\right)_{3}\right]$ [3], and the Prussian blue analogue imidazolium potassium hexacyanoferrate, $\left(\mathrm{C}_{3} \mathrm{H}_{5} \mathrm{~N}_{2}\right)_{2} \mathrm{~K}\left[\mathrm{Fe}(\mathrm{CN})_{6}\right]$. Using the reverse Monte Carlo algorithm, we are able to reconstruct the instantaneous local host-guest structural relationships, even in the disordered phases, in unprecedented detail. Our results demonstrate that the cation motion in both materials is heavily influenced by local distortions of the framework, which stabilise particular cation orientations. The instantaneous snapshots of these materials' local structure are corroborated by quasi-elastic neutron scattering measurements of the cation rotation dynamics.

[1] Kieslich, G. et al. (2014). Chem Sci. 5, 4712.

[2] Evans, N. L. et al. (2016), J. Am. Chem. Soc. 138, 9393.

[3] Duncan, H. D. et al. (2016). Dalton Trans. 45, 4380.
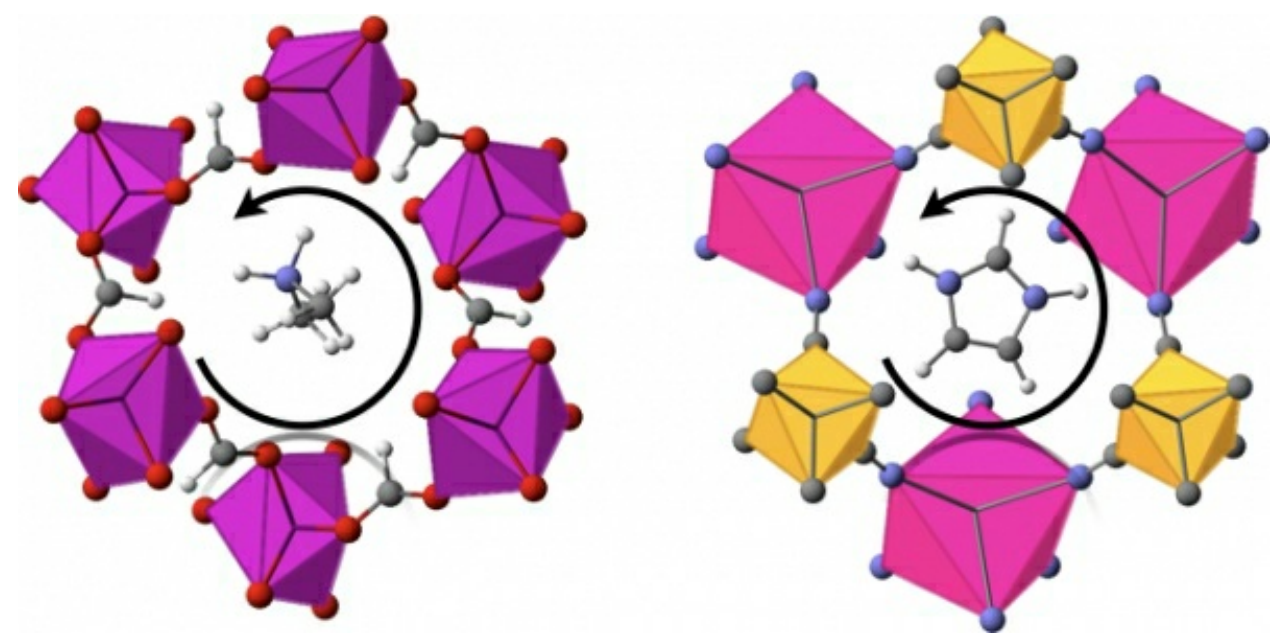

Keywords: framework materials, total neutron scattering, reverse Monte Carlo

Acta Cryst. (2017). A73, C71 